\title{
Sepsis and AKI in ICU Patients: The Role of Plasma Biomarkers
}

\author{
Paolo Lentini, ${ }^{1,2}$ Massimo de Cal, ${ }^{2,3}$ Anna Clementi, ${ }^{3}$ Angela D'Angelo, ${ }^{2}$ and Claudio Ronco ${ }^{3}$ \\ ${ }^{1}$ Department of Nephrology, San Bassiano Hospital, 36061 Bassano del Grappa, Italy \\ ${ }^{2}$ Division of Nephrology, University of Padua, 35100 Padua, Italy \\ ${ }^{3}$ Department of Nephrology, San Bortolo Hospital, 36100 Vicenza, Italy \\ Correspondence should be addressed to Paolo Lentini, paolo.lentini@yahoo.it
}

Received 3 August 2011; Revised 6 October 2011; Accepted 22 November 2011

Academic Editor: Alain Broccard

Copyright (C) 2012 Paolo Lentini et al. This is an open access article distributed under the Creative Commons Attribution License, which permits unrestricted use, distribution, and reproduction in any medium, provided the original work is properly cited.

Given the higher mortality rate of ICU patients with sepsis and AKI, we decided to investigate the possible correlation between serum biomarkers of organ damage, and endotoxin activity in ICU septic patients. Ninety-eight consecutive adult patients were enrolled in this study. Patients were divided in two groups depending on the presence of sepsis. Fifty-six patients had sepsis, while forty-two patients were nonseptic. Among septic patients, twenty-four subjects developed AKI, while thirty-two did not. AKI occurred in fourteen patients without sepsis as well. The levels of NGAL, BNP, and AOPP were significantly higher among septic patients compared with nonseptic subjects $(P<0.001)$. Among septic patients, subjects who developed AKI showed significant higher levels of NGAL and AOPP $(P=0.0425)$ and BNP $(P=0.0327)$. Among patients who developed AKI, a significant difference was found only in terms of AOPP levels between septic and nonseptic patients. The correlation between endotoxin activity and BNP in septic patients and the increase in the levels of NGAL, BNP, and AOPP in case of sepsis and AKI, in particular if they are associated, indicate a multiorgan involvement in these two conditions.

\section{Introduction}

Sepsis, defined as a systemic inflammatory response syndrome (SIRS) associated with an infectious disease [1,2], is a primary cause of morbidity and mortality in ICU [3] and critically ill patients. Mortality rates range from $20 \%$ for sepsis, to $40 \%$ for severe sepsis, to $60 \%$ for septic shock in ICU patients [4].

Gram-negative bacteria are implicated in $50-60 \%$ of sepsis, with Gram-positive bacteria accounting for a further $35-40 \%$ of cases. The remainder of causes are due to the less common causes of fungi, viruses, and protozoa [5].

The heat-stable toxic component of Gram-negative bacteria, identified for the first time by Pfeiffer at the end of the 19th century $[6,7]$ and called "endotoxin", is considered to play an important role in the pathogenesis of septic shock [8]. It causes the release of different cytokines, such as interleukin-1 (IL-1) and tumor necrosis factor- $\alpha$ (TNF$\alpha$ ), and interacts with the complement pathway and the coagulation system $[8,9]$.

Sepsis is also a contributing factor in more than $20 \%$ of cases of acute kidney injury (AKI) in ICU patients, with cases severe enough to require renal replacement therapy [10-12]. AKI occurs in $35-65 \%$ of ICU admissions, and most studies show a threefold to fivefold increase in the risk of death among patients with AKI compared to patients without AKI.

Given the higher mortality rate of ICU patients with sepsis and AKI, we decided to investigate the possible correlation between serum biochemical markers of organ damage, such as neutrophil gelatinase-associated lipocalin (NGAL), advanced oxidation protein products (AOPP), and brain natriuretic peptide (BNP) and endotoxin activity in ICU septic patients. Moreover, comparisons of the levels of these biomarkers were made between septic and nonseptic patients, septic patients with or without AKI, and between patients who developed AKI with or without sepsis.

\section{Material and Methods}

2.1. Study Population. Ninety-eight consecutive adult patients, admitted to ICU of San Bortolo Hospital, Vicenza, Italy, between October 2008 and august 2010, were enrolled in this study. Patients were divided in two groups depending 
TABLE 1: Clinical and biochemical characteristics of septic patients.

\begin{tabular}{|c|c|}
\hline & Septic patients $(N=56)$ \\
\hline Male sex $(\%)$ & 33.9 \\
\hline Age (years) & $69(48.7$ to 74.2$)$ \\
\hline Serum creatinine $(\mathrm{mg} / \mathrm{dL})$ & $1.64(1.04$ to 2.97$)$ \\
\hline Temperature $\left({ }^{\circ}\right.$ Celsius $)$ & $36.4 \pm 2.0$ \\
\hline WBC (million cells/mcL) & $12.3 \pm 9.4$ \\
\hline Platelets $\left(10^{3} / \mu \mathrm{L}\right)$ & $144.6 \pm 110.9$ \\
\hline $\mathrm{pH}$ & $7.359 \pm 0.154$ \\
\hline $\mathrm{Na}(\mathrm{mmol} / \mathrm{L})$ & $139.5 \pm 6.1$ \\
\hline $\mathrm{K}(\mathrm{mmol} / \mathrm{L})$ & $4.2 \pm 1.1$ \\
\hline $\mathrm{PaO}_{2} / \mathrm{FiO}_{2}(\mathrm{mmHg})$ & $206.4 \pm 112.5$ \\
\hline Sofa score & $10(8$ to 12$)$ \\
\hline Died (\%) & 32.1 \\
\hline
\end{tabular}

WBC: white blood cells; SOFA score: sequential organ failure assessment.

on the presence of sepsis, defined as systemic inflammatory response syndrome (SIRS) associated with an infectious process. SIRS was considered to be present when at least two of the following criteria were present: temperature above $38^{\circ} \mathrm{C}$ or below $36^{\circ} \mathrm{C}$, heart rate above 90 beats $/ \mathrm{min}$, respiratory rate above 20 breaths/min or partial pressure of carbon dioxide below $32 \mathrm{mmHg}$, and white blood cell count above $12,000 \mathrm{~mm}^{3}$ or below $4,000 \mathrm{~mm}^{3}$. Fifty-six patients had sepsis, while forty-two patients were nonseptic. Clinical and biochemical characteristic of septic patients are summarized in Table 1.

Among septic patients, twenty-four subjects developed AKI, defined by RIFLE criteria, while thirty-two did not. AKI occurred in fourteen patients without sepsis as well.

Within four hours after admission blood samples were taken for EAA (endotoxin activity assay), NGAL, and BNP measurement. EDTA was used as an anticoagulant. Heparinized blood samples were collected for AOPP evaluation.

Correlation between NGAL, AOPP, BNP and endotoxin activity in septic patients was evaluated. Moreover, comparisons of the levels of these biomarkers were made between septic and non septic patients, septic patients with or without AKI, and between patients who developed AKI with or without sepsis.

2.2. Endotoxin Activity Assay (EAA). Serum endotoxin activity was measured by the EAA ${ }^{\mathrm{tm}}$ which measures the degree of chemiluminescence of the circulating neutrophil population induced by the exposure to endotoxin.

The test is based on the interaction between the endotoxin and a specific antiendotoxin antibody. Complement components opsonize the endotoxin-antibody complex. The opsonized immune complex primes neutrophils in the blood to enhance their respiratory burst in response to zymosan. The respiratory burst of the neutrophils yields oxidants that react with luminal in the reaction mixture to emit chemiluminescence.

The chemiluminescence can then be detected in a photon-counting luminometer (SmartLine TL, Berthold Detection Systems, Pforzheim, Germany).
A basal activity measurement (Tube 1) in the absence of the specific antiendotoxin antibody measures the nonspecific oxidative burst of the patient's neutrophils. An additional control measurement including the specific antiendotoxin antibody and an excess of exogenous endotoxin (Tube 3) measures the maximum oxidative burst of the patient's neutrophils. The test measurement (Tube 2) includes the specific antibody to measure the neat level of endotoxin activity. The $\mathrm{EAA}^{\mathrm{tm}}$ level is calculated by normalizing the chemiluminescence in the test sample (Tube 2) against the maximum chemiluminescence (Tube 3), correcting both measurements for the basal activity chemiluminescence (Tube 1).

Endotoxin activity levels are expressed as units on a scale ranging from 0 to 1

0.00-0.39: EAA $^{\text {tm }}$ units: low endotoxin activity level,

0.40-0.59: EAA $^{\text {tm }}$ units: intermediate endotoxin activity level,

$\geq 0.60$ : EAA $^{\text {tm }}$ units: high endotoxin activity level.

2.3. NGAL and BNP Measurement. Plasma samples for NGAL and BNP measurement were stored at minus 80 degrees Celsius to be analyzed subsequently. Plasma NGAL and BNP were measured with fluorescence-based immunoassay with the Triage point-of-care analyzer (Biosite Inc., San Diego, CA, USA), which allows a rapid quantitative measurement of NGAL and BNP concentration in EDTAanticoagulated whole blood or plasma. NGAL and BNP concentrations were expressed as nanograms per millilitre $(\mathrm{ng} / \mathrm{mL})$ and pictograms per millilitre $(\mathrm{pg} / \mathrm{mL})$, respectively.

2.4. AOPP Measurement. AOPP levels were measured by spectrophotometry and calibrated with Chloramine-T solutions (Sigma Chemical Co., St. Louis, MO, USA), which adsorb at $340 \mathrm{~nm}$ in presence of potassium iodide. Two hundred microliters of plasma diluted $1 / 5$ in PBS, and $20 \mu \mathrm{L}$ of acetic acid were mixed and calibrated versus the standard reference of $200 \mu \mathrm{L}$ Chloramine-T solution $(0-100 \mu \mathrm{mol} / \mathrm{L})$ with $20 \mu \mathrm{L}$ of acetic acid and $10 \mu \mathrm{L}$ of potassium iodide.

The absorbance of the reaction mixture was read at $340 \mathrm{~nm}$ against a blank containing $200 \mu \mathrm{L}$ of PBS, $10 \mu \mathrm{L}$ of potassium iodide, and $20 \mu \mathrm{L}$ of acetic acid. AOPP concentrations were expressed as micromoles per liter of chloramine- $\mathrm{T}$ equivalents $(\mu \mathrm{mol} / \mathrm{L})$.

2.5. Statistical Analysis. Statistical analysis was performed with the use of SPSS software version 15.0. Categorical variables were expressed as percentages; continuous variables were expressed as means \pm standard deviation (parametric variables) or median (interquartile range; nonparametric variable). Differences between groups were analyzed using Student $t$-test and Mann-Whitney test as appropriate. Correlation was performed with the use of the Spearman rank coefficient. Two-tailed probability values of $<0.05$ were considered statistically significant. 
TABLE 2: Comparison of biochemical markers between septic patients and nonseptic patients.

\begin{tabular}{lccc}
\hline & Septic pts $(N=56)$ & Nonseptic pts $(N=42)$ & $P$ value \\
\hline Male sex $(\%)$ & 33.9 & 66.7 & 0.0013 \\
Age $($ years $)$ & $69(48.7$ to 74.2$)$ & $67(59$ to 75$)$ & 0.83 \\
Creatinine $(\mathrm{mg} / \mathrm{dL})$ & $1.64(1.04$ to 2.97$)$ & $1.0(0.8$ to 1.0$)$ & $<0.001$ \\
NGAL $(\mathrm{ng} / \mathrm{mL})$ & $459(213$ to 744$)$ & $115.7(79.2$ to 181.7$)$ & $<0.001$ \\
AOPP $(\mu \mathrm{mol} / \mathrm{L})$ & $505.1(307.6$ to 643.5$)$ & $135(61$ to 275$)$ & $<2$ to 174$)$ \\
BNP $(\mathrm{pg} / \mathrm{mL})$ & $409(212$ to 673$)$ & $5(4$ to 5$)$ & $<0.001$ \\
Sofa score & $10(8$ to 12$)$ & 16.7 & $<0.001$ \\
Died $(\%)$ & 32.1 & 0.08 \\
\hline
\end{tabular}

NGAL: neutrophil gelatinase-associated lipocalin; AOPP: advanced oxidation protein products; BNP: brain natriuretic peptide; SOFA score: sequential organ failure assessment.

TABLE 3: Comparison of biochemical markers between AKI and No-AKI septic patients.

\begin{tabular}{lccc}
\hline & AKI septic pts $(N=24)$ & No-AKI septic pts $(N=32)$ & $P$ value \\
\hline Male sex $(\%)$ & 29.2 & 37.5 & 0.51 \\
Age $($ years $)$ & $69(50$ to 71$)$ & $69(45$ to 76$)$ & 0.63 \\
Creatinine $(\mathrm{mg} / \mathrm{dL})$ & $2.3(1.5$ to 3.4$)$ & $321(154$ to 573$)$ & 0.8 to 1.9$)$ \\
NGAL $(\mathrm{ng} / \mathrm{mL})$ & $572(308$ to 819$)$ & $419.5(286.8$ to 607.4$)$ & 0.0065 \\
AOPP $(\mu \mathrm{mol} / \mathrm{L})$ & $554.0(366.8$ to 717.6$)$ & $348(174$ to 538$)$ & 0.0425 \\
BNP $(\mathrm{pg} / \mathrm{mL})$ & $576(291$ to 1723$)$ & $9(7$ to 12$)$ & 0.0327 \\
Sofa score & $11(8$ to 13$)$ & 21.9 & 0.28 \\
Died $(\%)$ & 45.8 & 0575 \\
\hline
\end{tabular}

NGAL: neutrophil gelatinase-associated lipocalin; AOPP: advanced oxidation protein products; BNP: brain natriuretic peptide; SOFA score: sequential organ failure assessment.

\section{Results}

Septic patients were divided in three groups depending on EAA levels. EAA < 40: 8 patients; EAA 40-60: 17 patients; EAA $>$ 60: 31 patients.

A significant correlation $(P=0.02)$ was found only between endotoxin activity and BNP levels of septic patients (Figure 1). The levels of NGAL, BNP, and AOPP were significantly higher among septic patients compared with nonseptic subjects $(P<0.001)$ (Table 2$)$. Among septic patients, subjects who developed AKI showed significant higher levels of NGAL and AOPP $(P=0.0425)$ and BNP $(P=0.0327)$ (Table 3$)$. Among patients who developed AKI, a significant difference was found only in terms of AOPP levels between septic and non septic patients (Table 4).

\section{Discussion}

As reported by Marshall et al., intermediate and high levels of endotoxin activity are often found in ICU septic patients [13], and they seem to correlate with the severity of the disease, in particular with the hemodynamic alterations [14]. Sepsis, indeed, frequently causes cardiac abnormalities and kidney dysfunction $[15,16]$ and, for this reason, can be considered as an important cause of type 5 cardiorenal syndrome [17].

In this study we investigated the possible correlation between endotoxin activity in septic ICU patients and biochemical markers of organ damage, such as NGAL, AOPP, and BNP.

As shown in Figure 1, a significant correlation was found only between endotoxin activity and BNP levels of septic patients $(P=0.02)$. BNP is considered to be a good diagnostic and prognostic biomarker, especially among patients with congestive heart failure [18]. Elevated levels of BNP are independent predictors of cardiovascular morbidity and mortality, both in patients with normal and impaired renal function, thus emphasizing the value of BNP in the assessment of cardiorenal syndrome [19]. In our study, intermediate and higher levels of endotoxin activity, which predict an elevated risk for developing severe sepsis, were associated with higher levels of BNP, which result from cardiac dysfunction induced by sepsis.

We also compared the levels of NGAL, AOPP, and BNP between septic and non septic patients, septic patients with or without AKI, and between patients who developed AKI with or without sepsis.

Serum NGAL has been shown to increase before serum creatinine in case of acute kidney injury [20] and has therefore become a novel early biomarker of acute renal damage [21]. Moreover, it was found to rise in patients with congestive heart failure, thus indicating a link between cardiac dysfunction and renal injury $[18,22,23]$.

Critically ill patients also present increased levels of AOPP, induced by the overproduction of reactive oxygen species (ROS) and the subsequent depletion of the antioxidant endogenous stores. AOPP levels were demonstrated to 
TABLE 4: Comparison of biochemical markers between AKI septic patients and AKI nonseptic patients.

\begin{tabular}{lccc}
\hline & AKI septic pts $(N=24)$ & AKI nonseptic pts $(N=14)$ & $P$ value \\
\hline Male sex $(\%)$ & 29.2 & 78.6 & 0.0033 \\
Age $($ years $)$ & $69(50$ to 71$)$ & $1.0(09$ to 80$)$ & 0.8 to 1.6$)$ \\
Creatinine $(\mathrm{mg} / \mathrm{dL})$ & $2.3(1.5$ to 3.4$)$ & $312(141$ to 633$)$ & $<067$ \\
NGAL $(\mathrm{ng} / \mathrm{mL})$ & $572(308$ to 819$)$ & $118.9(90.1$ to 152.5$)$ & 0.15 \\
AOPP $(\mu \mathrm{mol} / \mathrm{L})$ & $554.0(366.8$ to 717.6$)$ & $305(134$ to 559$)$ & 0.001 \\
BNP $(\mathrm{pg} / \mathrm{mL})$ & $576(291$ to 1723$)$ & $5(5$ to 5$)$ & $<0.001$ \\
Sofa score & $11(8$ to 13$)$ & 42.9 & 0.001 \\
Died $(\%)$ & 45.8 & & 0.85 \\
\hline
\end{tabular}

NGAL: neutrophil gelatinase-associated lipocalin; AOPP: advanced oxidation protein products; BNP: brain natriuretic peptide; SOFA score: sequential organ failure assessment.

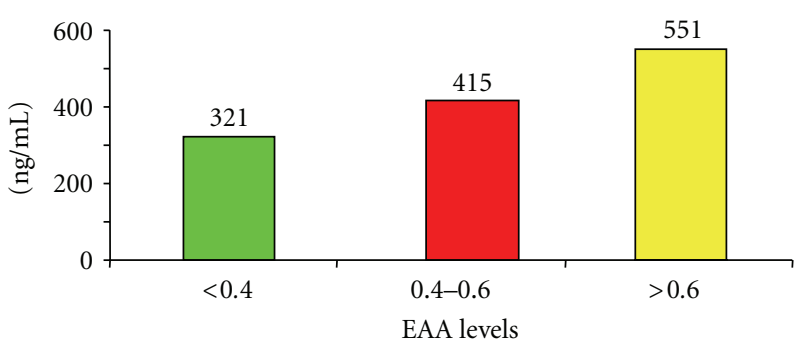

(a) NGAL

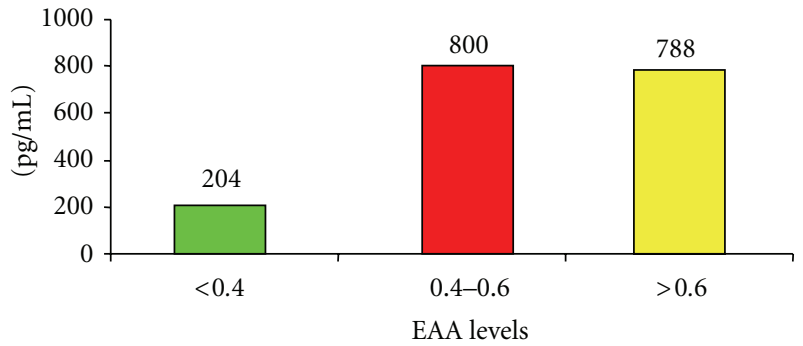

(b) BNP, $P=0.02$

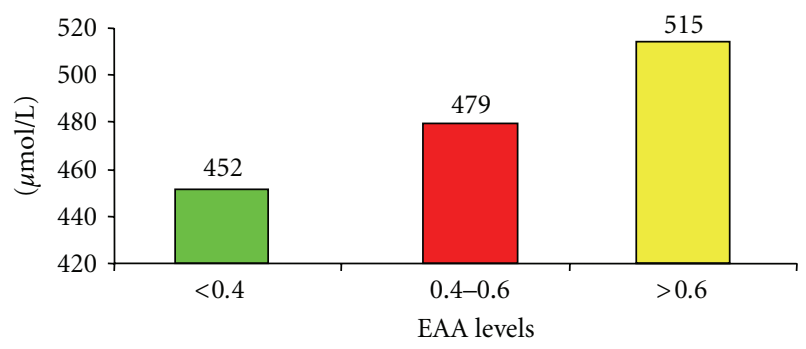

(c) AOPP

Figure 1: Correlation between EAA $(<0.40 ; 0.40-0.60 ;>0.60$ units $)$ and the levels of NGAL, BNP, and AOPP.

correlate with the risk to develop severe sepsis and with the severity of AKI in ICU patients [19,24].

In our study, the levels of NGAL, BNP, and AOPP were significantly higher among septic patients compared with non septic subjects $(P<0.001)$, as shown in Table 2 . Among septic patients, subjects who developed AKI showed significant higher levels of NGAL and AOPP $(P=0.0425)$ and BNP $(P=0.0327)$ (Table 3$)$. Among patients who developed AKI, a significant difference was found only in terms of AOPP levels between septic and non septic patients (Table 4).

These data suggest that sepsis and AKI are responsible for the increase in the level of the three biomarkers, in particular if they are associated. When limiting to the AKI patients, there was no significant difference in terms of NGAL and BNP levels between septic and non septic patients. The reason for this finding remains to be clarified. A possible explanation could be that renal damage alone can cause a similar increase in the level of the two biomarkers, independently on the presence of sepsis.

\section{Conclusions}

In septic ICU patients endotoxin activity correlates with BNP levels. NGAL, AOPP, and BNP levels seem to be higher in patients with sepsis and AKI, in particular if they are associated. In case of AKI, a significant difference between septic and nonseptic patients was found only for AOPP levels.

NGAL, AOPP, and BNP increase in case of sepsis, thus indicating both cardiac and renal impairment. For this reason, the rise in their levels in this condition can allow clinicians to individualize patients at higher risk for developing severe sepsis and therefore at higher risk of death.

\section{References}

[1] M. M. Levy, M. P. Fink, J. C. Marshall et al., “2001 SCCM/ESICM/ACCP/ATS/SIS international sepsis definitions conference," Critical Care Medicine, vol. 31, no. 4, pp. 12501256, 2003. 
[2] R. C. Bone, R. A. Balk, F. B. Cerra et al., "Definitions for sepsis and organ failure and guidelines for the use of innovative therapies in sepsis. The ACCP/SCCM Consensus Conference Committee. American College of Chest Physicians/Society of Critical Care Medicine," Chest, vol. 101, no. 6, pp. 1644-1655, 1992.

[3] G. S. Martin, D. M. Mannino, S. Eaton, and M. Moss, "The epidemiology of sepsis in the United States from 1979 through 2000," New England Journal of Medicine, vol. 348, no. 16, pp. 1546-1554, 2003.

[4] C. Brun-Buisson, "The epidemiology of the systemic inflammatory response," Intensive Care Medicine, vol. 26, no. 1, pp. S64-S74, 2000.

[5] R. L. Paterson and N. R. Webster, "Sepsis and the systemic inflammatory response syndrome," Journal of the Royal College of Surgeons of Edinburgh, vol. 45, no. 3, pp. 178-182, 2000.

[6] H. Brade, L. Brade, U. Schade et al., "Structure, endotoxicity, immunogenicity and antigenicity of bacterial lipopolysaccharides (endotoxins, O-antigens)," Progress in Clinical and Biological Research, vol. 272, pp. 17-45, 1988.

[7] R. Pfeiffer, "Untersuchungen über das Choleragift," Zeitschrift für Hygiene und Infektionskrankheiten, vol. 11, no. 1, pp. 393$412,1892$.

[8] D. N. Cruz, R. Bellomo, and C. Ronco, "Clinical effects of polymyxin B-immobilized fiber column in septic patients," Contributions to Nephrology, vol. 156, pp. 444-451, 2007.

[9] U. N. Das, "Critical advances in septicemia and septic shock," Critical Care, vol. 4, no. 5, pp. 290-296, 2000.

[10] S. M. Bagshaw, S. Uchino, R. Bellomo et al., "Septic acute kidney injury in critically ill patients: clinical characteristics and outcomes," Clinical Journal of the American Society of Nephrology, vol. 2, no. 3, pp. 431-439, 2007.

[11] E. A. J. Hoste, N. H. Lameire, R. C. Vanholder, D. D. Benoit, J. M. A. Decruyenaere, and F. A. Colardyn, "Acute renal failure in patients with sepsis in a surgical ICU: predictive factors, incidence, comorbidity, and outcome," Journal of the American Society of Nephrology, vol. 14, no. 4, pp. 1022-1030, 2003.

[12] J. A. Lopes, S. Jorge, C. Resina et al., "Acute renal failure in patients with sepsis," Critical Care, vol. 11, article no. 411, 2007.

[13] J. C. Marshall, P. M. Walker, D. M. Foster et al., "Measurement of endotoxin activity in critically ill patients using whole blood neutrophil dependent chemiluminescence," Critical Care, vol. 6, no. 4, pp. 342-348, 2002.

[14] G. Monti, M. Bottiroli, G. Pizzilli et al., "Endotoxin activity level and septic shock: a possible role for specific antiendotoxin therapy?" Contributions to Nephrology, vol. 167, pp. 102-110, 2010.

[15] J. Charpentier, C. E. Luyt, Y. Fulla et al., "Brain natriuretic peptide: a marker of myocardial dysfunction and prognosis during severe sepsis," Critical Care Medicine, vol. 32, no. 3, pp. 660-665, 2004.

[16] S. M. Bagshaw, C. George, and R. Bellomo, "Early acute kidney injury and sepsis: a multicentre evaluation," Critical Care, vol. 12, no. 2, article no. R47, 2008.

[17] C. Ronco, M. Haapio, A. A. House, N. Anavekar, and R. Bellomo, "Cardiorenal syndrome," Journal of the American College of Cardiology, vol. 52, no. 19, pp. 1527-1539, 2008.

[18] A. Maisel and C. Mueller, "State of the art: using natriuretic peptide levels in clinical practice," European Journal of Heart Failure, vol. 10, no. 9, pp. 824-839, 2008.

[19] C. Bruch, C. Fischer, J. Sindermann, J. Stypmann, G. Breithardt, and R. Gradaus, "Comparison of the prognostic usefulness of $\mathrm{N}$-terminal pro-brain natriuretic peptide in patients with heart failure with versus without chronic kidney disease," American Journal of Cardiology, vol. 102, no. 4, pp. 469-474, 2008.

[20] J. M. Thurman and C. R. Parikh, "Peeking into the black box: new biomarkers for acute kidney injury," Kidney International, vol. 73, no. 4, pp. 379-381, 2008.

[21] V. S. Vaidya, M. A. Ferguson, and J. V. Bonventre, "Biomarkers of acute kidney injury," Annual Review of Pharmacology and Toxicology, vol. 48, pp. 463-493, 2008.

[22] K. Damman, D. J. van Veldhuisen, G. Navis, A. A. Voors, and H. L. Hillege, "Urinary neutrophil gelatinase associated lipocalin (NGAL), a marker of tubular damage, is increased in patients with chronic heart failure," European Journal of Heart Failure, vol. 10, no. 10, pp. 997-1000, 2008.

[23] B. Poniatowski, J. Malyszko, H. Bachorzewska-Gajewska, J. S. Malyszko, and S. Dobrzycki, "Serum neutrophil gelatinaseassociated lipocalin as a marker of renal function in patients with chronic heart failure and coronary artery disease," Kidney and Blood Pressure Research, vol. 32, no. 2, pp. 77-80, 2009.

[24] P. Lentini, M. de Cal, D. Cruz et al., "The role of advanced oxidation protein products in intensive care unit patients with acute kidney injury," Journal of Critical Care, vol. 25, no. 4, pp. 605-609, 2010. 


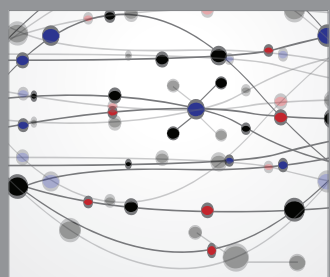

The Scientific World Journal
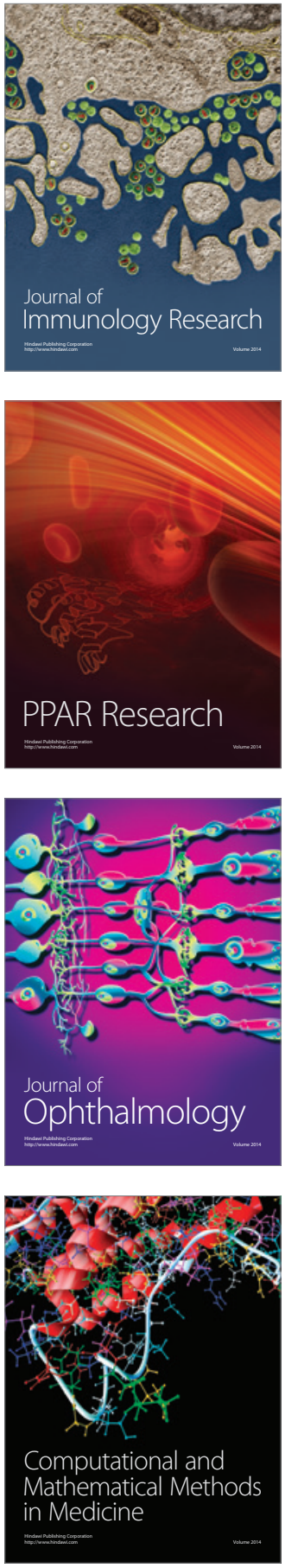

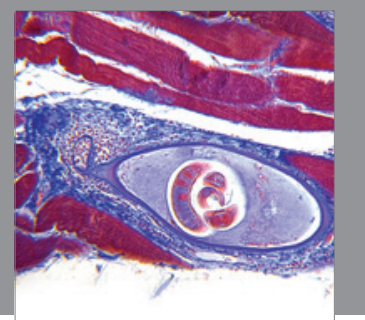

Gastroenterology

Research and Practice
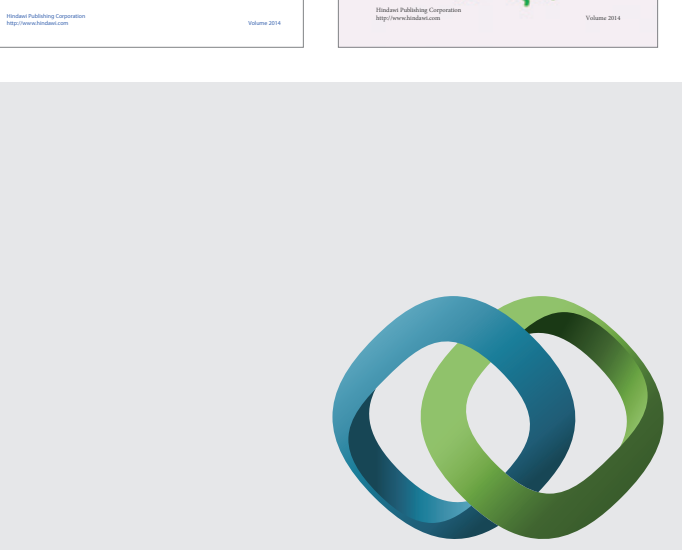

\section{Hindawi}

Submit your manuscripts at

http://www.hindawi.com
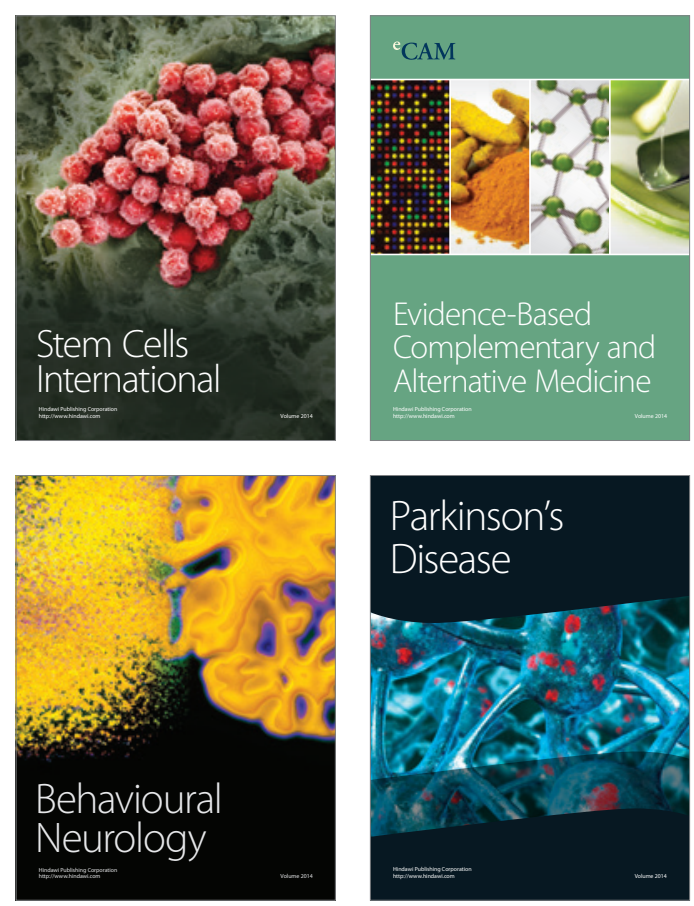

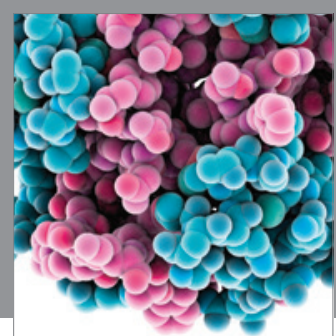

Journal of
Diabetes Research

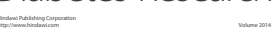

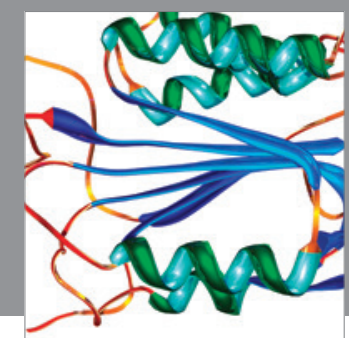

Disease Markers
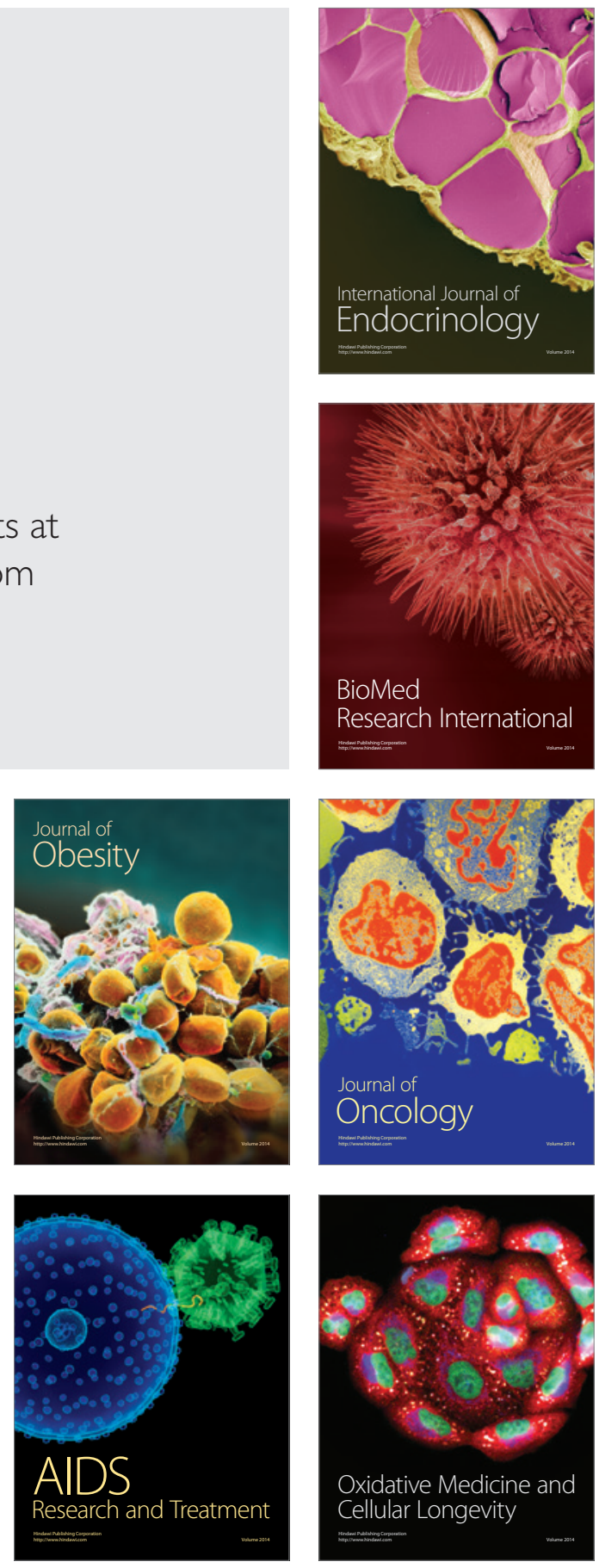